\title{
Comparative Evaluation of Antioxidant Enzymes and Serum Selenium Levels in Libyan Atherosclerotic Patients
}

\author{
Rabia Alghazeer ${ }^{*}$, Eenas Aboulmeedah ${ }^{1}$, Sana Elgahmasi', Nadia Alghazir ${ }^{2}$, \\ Zohra Almukthar ${ }^{3}$, Maryouma Enaami ${ }^{4}$, Abdurrahman Rhuma ${ }^{1}$ \\ ${ }^{1}$ Department of Chemistry, Faculty of Sciences, University of Tripoli, Tripoli, Libya \\ ${ }^{2}$ Pediatric Department, Faculty of Medicine, University of Tripoli, Tripoli, Libya \\ ${ }^{3}$ Tajoura National Heart Center, Tripoli, Libya \\ ${ }^{4}$ Department of Statistics, Faculty of Science, University of Tripoli, Tripoli, Libya \\ Email: *R.alghazeer@uot.edu.ly, *Rabia_Alghazeer@yahoo.com
}

How to cite this paper: Alghazeer, R., Aboulmeedah, E., Elgahmasi, S., Alghazir, N., Almukthar, Z., Enaami, M. and Rhuma, A. (2019) Comparative Evaluation of Antioxidant Enzymes and Serum Selenium Levels in Libyan Atherosclerotic Patients. Journal of Biosciences and Medicines, 7, 51-69.

https://doi.org/10.4236/jbm.2019.77005

Received: June 5, 2019

Accepted: July 12, 2019

Published: July 15, 2019

Copyright $\odot 2019$ by author(s) and Scientific Research Publishing Inc. This work is licensed under the Creative Commons Attribution International License (CC BY 4.0).

http://creativecommons.org/licenses/by/4.0/

\begin{abstract}
Introduction: Atherosclerosis is the major source of morbidity and mortality in the developed countries like Libya. Oxidative stress represents a risk factor and plays a key role at several steps of atherosclerosis. Formation of atherosclerotic plaques is the main reason for coronary artery disease. Aim: This study is aimed to assess the impact of the imbalance between body oxidants and antioxidants (oxidative stress) and its involvement in developing and severity of atherosclerosis in atherosclerotic with and without diabetes Libyan patients. Methods: The study sample comprised of atherosclerosis with and without diabetes patients $(n=73)$, as well as healthy controls $(n=27)$. Blood samples were collected to determine the levels of malonaldehyde (MDA) as oxidative biomarker, serum selenium level, total antioxidant status (TAS) level and some antioxidant enzymes including glutathione peroxidase (GPx), Catalase (Cat) and superoxide dismutase (SOD). Additionally, oxidant/antioxidant status was compared between atherosclerotic patients with and without diabetes in comparison to controls. Results: The results indicated a significant increase in MDA level among atherosclerotic patients compared to healthy subjects $(\mathrm{P}<0.05)$. While the level of TAS, Cat, SOD and GPx was significantly decreased among patients compared to the controls $(\mathrm{P}<0.05)$. For the atherosclerotic diabetic patients, the study was found a rise in the level of MDA as well as a marked decrease in TAS and the activity of tested antioxidant enzymes $(\mathrm{P}<0.05)$. A good correlation was obtained between elevated MDA and decreased TAS, Cat, SOD and GPx. Conclusion: Determination of antioxidative defense markers contributes to understanding the effect of oxidative stress on the development and the prevention of cardiovascular disease.
\end{abstract}




\section{Keywords}

Atherosclerosis, Free Radicals, Oxidative Stress, Antioxidants

\section{Introduction}

Atherosclerosis is the major source of morbidity and mortality in many African countries including Libya [1]. It is characterized by the accumulation of cholesterol deposits in macrophages, which lead to a proliferation of certain cell types within the arterial wall (foam cells) that gradually impinges on the vessel lumen and impedes blood flow. Reduced blood flow to target organs like heart and brain can cause attack and stroke.

Oxidative stress is the result of imbalance between the reactive oxygen species (ROS) and antioxidants in the body. Oxidative modifications within the arterial wall that may initiate and/or contribute to atherogenesis are likely to occur, which result in imbalance between the reactive oxygen species and antioxidants in the body. Therefore, it is important to consider the sources of oxidants in the context of available antioxidants [2] [3]. Oxidative stress, particularly the oxidation of low density lipoprotein (LDL), represents a risk factor and plays a key role at several steps of atherosclerosis, according to the oxidative-modification hypothesis of atherosclerosis [4] [5]. The oxidative modification hypothesis, antioxidant protection of LDL in the extracellular space deserves focus, as oxidized LDL has many potential proatherogenic activities [6], and the cellular accumulation of oxidized LDL is considered a hallmark of atherosclerosis [7].

The possible sources of oxidative stress in atherosclerosis results from endothelial production of ROS, especially superoxide $\left(\mathrm{O}_{2}^{-}\right)$, with the subsequent reaction with nitric oxide (NO) are an important mechanism of vascular dysfunction in atherosclerosis. $\mathrm{O}_{2}^{-}$and $\mathrm{NO}$ rapidly interact to generate peroxynitrite $\left(\mathrm{ONOO}^{-}\right)$, a potent oxidant and a mediator of vascular tissue injury [8] [9]. Excess generation of ROS has been demonstrated in atherosclerosis and peroxynitrite formation has been shown to occur in atherosclerotic human [10] [11].

Diabetes mellitus (DM) has also been shown to play a crucial role in progress of cardiovascular diseases [12] [13]. Recently, it has been found that significant indicators for rising risk of coronary heart disease, stroke or death are associated with higher levels of baseline HbA1c [14] [15]. Actually, interest has developed in alternative markers, such as plasma markers of oxidative stress, which have a role to predict CVD risk [16].

Intracellular antioxidant defense is primarily provided by antioxidant enzymes which catalyze decomposition of reactive oxygen species. The three major antioxidant enzymes, superoxide dismutase (SOD), glutathione peroxidase (GPx), and catalase (Cat), differ from each other in structure, tissue distribution, and cofactor requirement. In addition, Selenium is a powerful antioxidant regu- 
lating the activity of the glutathione peroxidase enzymes which catalyze the detoxification of hydrogen peroxide and organic hydroperoxides [17]. Therefore, it is worthy to know whether the change in the level of antioxidants has an impact in the progressed of atherosclerosis.

\section{Patients and Methods}

\subsection{Study Design}

This study was conducted which recruited males with atherosclerosis and volunteers healthy subjects. A cross-sectional study was conducted on 73 atherosclerotic patients aged 45 - 65 years who attended the Tajora Central Heart Hospital. The control group (27 males) encompassed blood donors from the hospital staff with no known pathology that can be classified as their cases, their age was within the same age range as the other patients. A detailed medical history was taken and a physical examination was performed upon all participants. The Ethics committee of Biotechnology Research Center (Tripoli, Libya) approved the study (BEC-BTRC 03-2016). All patients provided a written informed consent prior to start of the study procedures.

\subsection{Selection Criteria}

Subjects included in the current study were selected according to the following criteria: first, they were newly diagnosed as atherosclerotic patient. Hemolytic anemia, hemoglobin variants, hepatic disease and infectious diseases like tuberculosis, sarcoidosis, etc., were excluded from this study. In addition, subjects under treatment with drugs such as chelating agents, ethambutol, and D-penicillamine were also excluded.

\subsection{Blood Sample Collection}

Blood samples were collected into commercial tubes after overnight fasting for analysis of laboratory parameters. Venous blood samples were obtained from the capital vein of each participant using sterile disposable plastic syringes. Specimens were collected at the same standardized time to minimize any effect of diurnal variation. After centrifugation, the clear, non-hemolyzed supernatant sera were separated using clean dry disposable plastic syringes. Samples were stored at $-80^{\circ} \mathrm{C}$ and used within 1 month for the analysis of oxidative and non-oxidative parameters.

\subsection{Determination of Biochemical Parameters}

Various biochemical parameters in blood were measured according to standard methods including Glucose, Glycosylated hemoglobin A1c (HbA1c), total protein, alanine amino transaminase (ALT), aspartate amino transaminase (AST). Triglycerides (TG), total cholesterol (TC), high-density lipoprotein cholesterol (HDL-C), and Low-density lipoprotein cholesterol (LDL-C). All parameters measured according to standard protocols in all subjects using Pre-modular 
analytics by using a kit with Cobas Integra ${ }^{\oplus} 400$ plus in the Biochemistry Laboratory, at the Tajora Central Heart Hospital, Tripoli, Libya.

\subsection{Oxidative Stress Parameter}

Thiobarbituric acid reacting substances (TBARS) content, a measure of lipid peroxidation was determined spectrophotometrically in serum according to the method of Zhang, [18].

\subsection{Antioxidative Parameters}

The catalase activity was assayed by the spectrophotometric method, based on the ability of hydrogen peroxide to form a stable stained complex with dichromate/acetic acid reagent [19]. Glutathione peroxidase activity was determined according to the method of Hafeman et al. [20]. Estimation of SOD activity was performed using a SOD Assay kit-WST (Sigma Aldrich, USA) according to the manufacturer's protocol (Dojindo, Gaithersburg, MD, USA). Total antioxidant activity was preformed using commercial tests manufactured by kit (Antioxidant assay kit, cat. No. CS0790; Sigma-Aldrich, St. Louis, MO, USA). The level of selenium was determined by using Inductively Coupled Plasma Optical Emission Spectrometer (ICP-OES) technique.

\subsection{Statistical Analysis}

Statistical analysis was done using the SPSS program version 11.0 statistic software package. The results were expressed as mean \pm standard deviation. Statistical significance was assessed using the analysis of variance (ANOVA), Tukey HSD and Pearson's correlation coefficient. $\mathrm{P}$ values of $<0.05$ were considered statistically significant.

\section{Results}

Figure 1 shows demographic data of atherosclerotic patients and control group. The clinical and biochemical data for the population studied are summarized in Table 1. Among 73 atherosclerotic male subjects, $43.84 \%$ were diabetic and 57.53\%

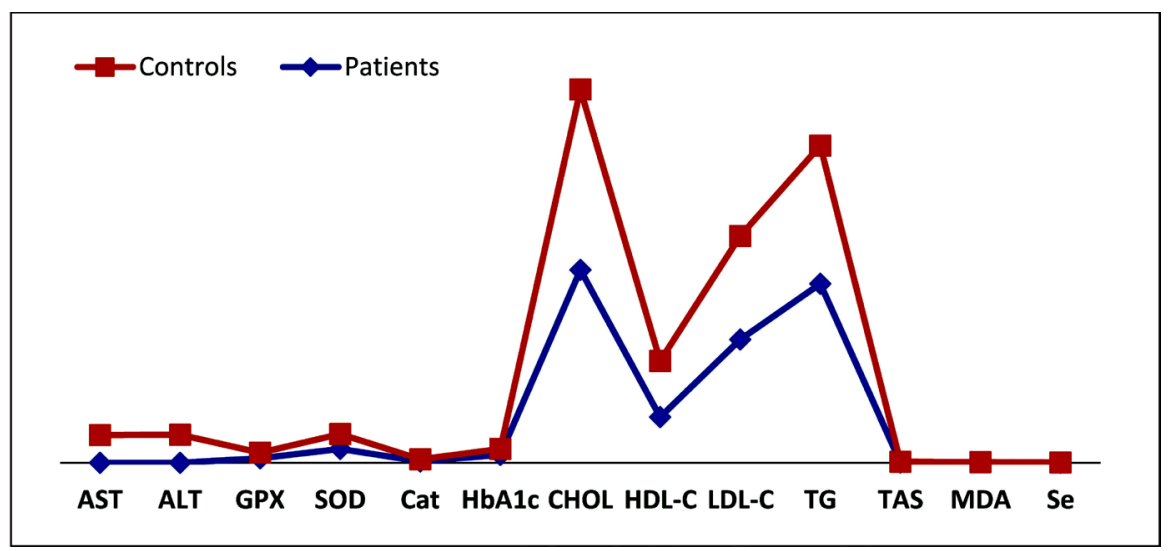

Figure 1. Demographic data of atherosclerotic patients and healthy subjects (controls). 
were hypertensive while 53.42\% were smokers. Body mass indexes (BMI) of patients were found significantly increased from control $(P<0.009) .57 .53 \%$ of patients were on antihypertensive treatment and their blood pressure was significantly higher compared to control Table 1.

Serum cholesterol and LDL concentrations in atherosclerotic subjects were significantly raised $(P<0.01, P<0.05)$ as compared to control. Serum total cholesterol (TC) concentration in-group with atherosclerosis was no significantly higher $(P>0.05)$ as compared to control (Table 2). However, no significant change was observed in group with atherosclerosis in comparison with control $(P>0.05)$ (Table 1).

\subsection{Patient Characteristics}

BMI, Body mass index, TC: Total cholesterol; TG: Triglyceride; LDL-C: Lowdensity lipoprotein cholesterol; HDL-C: High-density lipoprotein cholesterol, $\mathrm{HbA}_{1 \mathrm{c}}$ : Glycosylated hemoglobin; SOD: Superoxide dismutase; GPx: Glutathione peroxidase; MDA: Malondialdehyde; TAS: Total Antioxidants.

The mean percentage changes of the oxidant and antioxidant parameters in patients as compared to the controls are shown in (Figure 2). In comparison with control group, the level of MDA was increased significantly $(P<0.001)$ from $0.066 \pm 0.048$ to $0.52 \pm 0.32 \mathrm{nmol} / \mathrm{mgP}$ by $213 \%$ and a significant increase in HbA1c levels $(P<0.001)$ from $5.27 \% \pm 0.40 \%$ to $6.88 \pm 1.74 \%$ by $30.55 \%$ was observed in atheroserotic patients with respect to normal healthy control subjects (Table 1 and Figure 2). A significant decrease in Cat activity $(\mathrm{P}<0.002)$ by $3.98 \%$ (from $1.885 \pm 0.84 \mathrm{U} / \mathrm{mgP}$ to $1.81 \pm 0.90 \mathrm{U} / \mathrm{mg} \mathrm{P}$ ) was found in patients as compared with control groups (Table 1 and Figure 2).

Table 1 shows the distribution of serum selenium concentration in patients and controls. The mean concentration of serum selenium was marked decrease

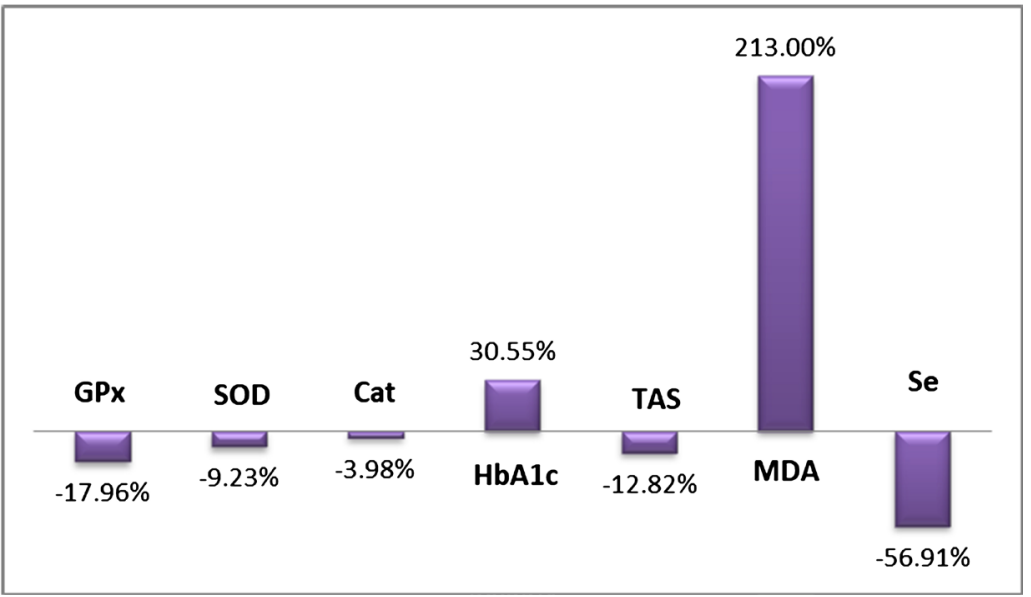

Values are expressed as mean \pm SD for $n$ subjects, HbA1c: Glycated haemoglobin A1c; SOD: Superoxide dismutase; GPx: Glutathione peroxidase; MDA: Malondialdehyde; TAS: Total Antioxidant status.

Figure 2. Percentage change of mean oxidant and antioxidant parameters in atherosclerotic patients compared to controls. 
$(P<0.00)$ by $56.91 \%$ (from $0.094 \pm 0.0114$ to $0.0405 \pm 0.0039 \mathrm{ppm}$ for controls and atherosclerotic subjects) (Table 1 and Figure 2).

Thirty-two atherosclerotic type-II diabetes mellitus and forty-one atherosclerotic non-diabetic age matched normal blood pressure controls were included in the study. Baseline characteristics of both groups are shown in Table 2. In type-II diabetes mellitus group, HbAlc was significantly higher than the non-diabetic group $(P<0.000)$ (Table 2$)$. Serum cholesterol, TG and LDL concentrations in diabetic subjects were significantly raised as compared to non-diabetic group (TC: $170.35 \pm 25.79 \mathrm{mg} / \mathrm{dl}$ vs. $162.7 \pm 35.8 \mathrm{mg} / \mathrm{dl}$, TG: 145.72 $\pm 85.31 \mathrm{mg} / \mathrm{dl}$ vs. $134.83 \pm 50.93 \mathrm{mg} / \mathrm{dl}$, LDL: $98.5 \pm 26.245 \mathrm{mg} / \mathrm{dl} \mathrm{vs} .93 .625 \pm$ $29.71 \mathrm{mg} / \mathrm{dl}$. However, there was no significant change was observed in the level of HDL AST, ALT in diabetic group with respect to the control group $(P>0.05)$.

Figure 3 shows the values of MDA and anti-oxidative parameters in atherosclerotic diabetic patients and atherosclerotic none diabetic subjects. The results

Table 1. Characteristic laboratory variables and the values of anti-oxidative parameters atherosclerotic and control subjects.

\begin{tabular}{ccccc}
\hline $\begin{array}{c}\text { Measure Indices } \\
\text { (unit) }\end{array}$ & Patients with & $\begin{array}{c}\text { Controls } \\
(\mathrm{n}=30)\end{array}$ & P value \\
\hline Age & $(\mathrm{Years}, \mathrm{X} \pm \delta)$ & $54.33 \pm 6.04$ & $48.52 \pm 5.03$ & 0.000 \\
BMI & $\left(\mathrm{Kg} / \mathrm{m}^{2}, \mathrm{X} \pm \delta\right)$ & $19.66 \pm 2.88$ & $16.96 \pm 2.71$ & 0.009 \\
Diabetes & $\mathrm{n}(\%)$ & $43.84 \%$ & - & \\
Hypertension & $\mathrm{n}(\%)$ & $57.53 \%$ & - & \\
Smoking & $\mathrm{n}(\%)$ & $53.42 \%$ & - & \\
AST & $(\mathrm{U} / \mathrm{l}, \mathrm{X} \pm \delta)$ & $25.38 \pm 10.75$ & $24.29 \pm 4.18$ & 0.664 \\
ALT & $(\mathrm{U} / \mathrm{l}, \mathrm{X} \pm \delta)$ & $26.77 \pm 13.44$ & $24.74 \pm 11.83$ & 0.632 \\
GPx & $(\mathrm{U} / \mathrm{mg} \mathrm{P}, \mathrm{X} \pm \delta)$ & $4.02 \pm 1.95$ & $4.9 \pm 2.73$ & 0.137 \\
SOD & $(\mathrm{U} / \mathrm{mg} \mathrm{P}, \mathrm{X} \pm \delta)$ & $12.09 \pm 5.5$ & $13.32 \pm 6.18$ & 0.537 \\
Cat & $(\mathrm{U} / \mathrm{mg} \mathrm{P}, \mathrm{X} \pm \delta)$ & $1.18 \pm 0.90$ & $1.885 \pm 0.84$ & 0.002 \\
HbAlc & $\%$ & $6.88 \pm 1.74$ & $5.27 \pm 0.40$ & 0.000 \\
TC & $(\mathrm{mg} / \mathrm{dl}, \mathrm{X} \pm \delta)$ & $170.21 \pm 45.01$ & $159.6 \pm 27.37$ & 0.015 \\
HDL-C & $(\mathrm{mg} / \mathrm{dl}, \mathrm{X} \pm \delta)$ & $40.30 \pm 9.7$ & $49.59 \pm 10.33$ & 0.000 \\
LDL-C & $(\mathrm{mg} / \mathrm{dl}, \mathrm{X} \pm \delta)$ & $108.7 \pm 28.65$ & $91.75 \pm 24.24$ & 0.047 \\
TG & $(\mathrm{mg} / \mathrm{dl}, \mathrm{X} \pm \delta)$ & $158.15 \pm 97.14$ & $122 \pm 52.2$ & 0.111 \\
TAS & $(\mathrm{nmol} / \mathrm{mgP}, \mathrm{X} \pm \delta)$ & $0.34 \pm 0.22$ & $0.39 \pm 0.20$ & 0.597 \\
MDA & $(\mathrm{nmol} / \mathrm{mgP}, \mathrm{X} \pm \delta)$ & $0.52 \pm 0.32$ & $0.166 \pm 0.048$ & 0.000 \\
Se & $(\mathrm{ppm}, \mathrm{X} \pm \delta)$ & $0.0405 \pm 0.0039$ & $0.094 \pm 0.0114$ & 0.000 \\
\hline
\end{tabular}

Values are expressed as mean \pm SD for $\mathrm{n}$ subjects, BMI, Body mass index, TC: Total cholesterol; TG: Triglyceride; LDL-C: Low-density lipoprotein cholesterol; HDL-C: High-density lipoprotein cholesterol, HbA1c: Glycated haemoglobin A1c; SOD: Superoxide dismutase; GPx: Glutathione peroxidase; MDA: Malondialdehyde; TAS: Total Antioxidant status. One-way ANOVA followed by Tuceky test, $\mathrm{n}=3$. Non-significant groups: $P \geq 0.05 ;{ }^{\star} P<0.05 ;{ }^{\star} P P 0.001$ considered statistically significant as compared to atherosclerotic non-diabetic group. 
Table 2. Characteristic laboratory variables and the values of anti-oxidative parameters atherosclerotic patients with and without type 2 diabetes.

\begin{tabular}{ccccc}
\hline & & $\begin{array}{c}\text { Diabetic patients } \\
(\mathbf{n}=32)^{*}\end{array}$ & $\begin{array}{c}\text { Non diabetic patients } \\
(\mathbf{n}=41)\end{array}$ & $P$ value \\
\hline Age & $($ years, $\mathrm{X} \pm \delta)$ & $56.47 \pm 5.41$ & $52.66 \pm 6.04$ & 0.013 \\
BMI & $\left(\mathrm{Kg} / \mathrm{m}^{2}, \mathrm{X} \pm \delta\right)$ & $20.11 \pm 2.58$ & \pm 3.0819 .32 & 0.981 \\
Diabetes duration & Years & $1.5 \pm 0.51$ & - & - \\
Hypertension & $\%$ & $50 \%$ & $63.41 \%$ & - \\
Smoking & $\%$ & $56.25 \%$ & $51.22 \%$ & - \\
HbAlc & $\%$ & $8.25 \pm 1.64^{* *}$ & $5.84 \pm 0.88$ & 0.000 \\
AST & $\mathrm{U} / \mathrm{L}$ & $24.35 \pm 8.72$ & $26.19 \pm 12.15$ & 0.632 \\
ALT & $\mathrm{U} / \mathrm{L}$ & $25.76 \pm 11.25$ & $27.57 \pm 15.014$ & 0.664 \\
TC & $(\mathrm{mg} / \mathrm{dl}, \mathrm{X} \pm \delta)$ & $170.35 \pm 25.79^{*}$ & $162.7 \pm 35.8$ & 0.026 \\
HDL-C & $(\mathrm{mg} / \mathrm{dl}, \mathrm{X} \pm \delta)$ & $40.16 \pm 8.65$ & $40.41 \pm 10.51$ & 0.993 \\
LDL-C & $(\mathrm{mg} / \mathrm{dl}, \mathrm{X} \pm \delta)$ & $98.5 \pm 26.245^{*}$ & $93.625 \pm 29.71$ & 0.052 \\
TG & $(\mathrm{mg} / \mathrm{dl}, \mathrm{X} \pm \delta)$ & $145.72 \pm 85.31^{*}$ & $134.83 \pm 50.93$ & 0.033 \\
\hline
\end{tabular}

Values are expressed as mean \pm SD for $\mathrm{n}$ subjects, BMI, Body mass index, ${ }^{*}$ : non-insulin dependent (Type-2) diabetes mellitus, TC: Total cholesterol; TG: Triglyceride; LDL-C: Low-density lipoprotein cholesterol; HDL C: High density lipoprotein cholesterol; $\mathrm{HbA}_{1 \mathrm{c}}$ : Glycosylated hemoglobin. One-way ANOVA followed by Tuckey test, $\mathrm{n}=3$. Non-significant groups: $P \geq 0.05$; ${ }^{*} P<0.05 ;{ }^{*} P<0.001$ considered statistically significant as compared to atherosclerotic non-diabetic group.

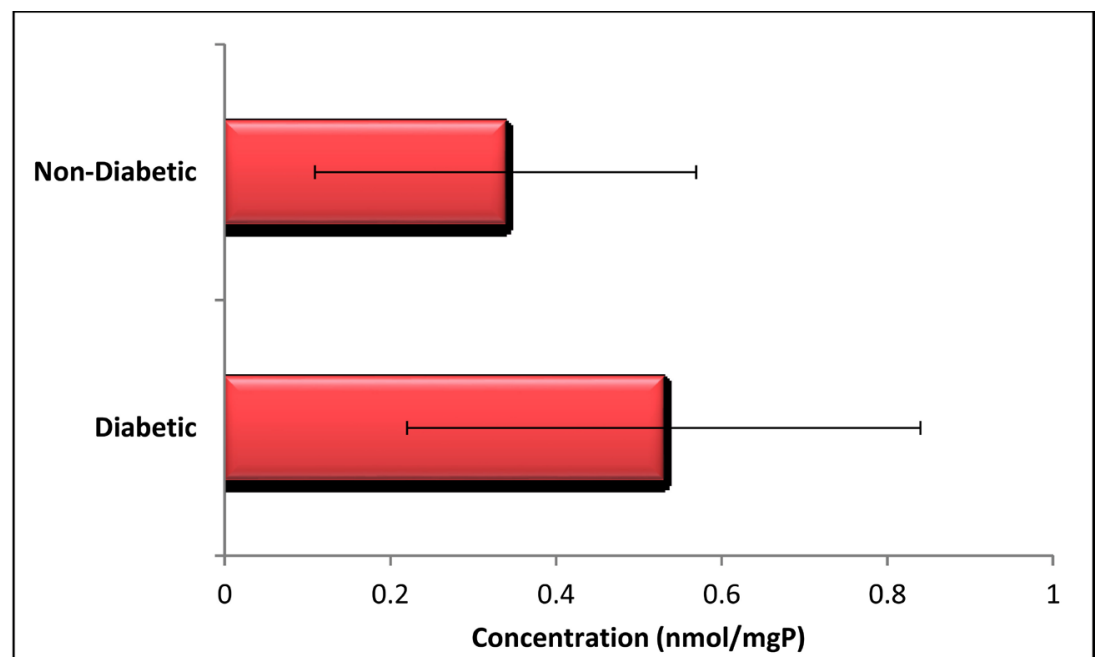

Values are expressed as mean \pm SD for $\mathrm{n}$ subjects, Diabitcs: non-insulin dependent (Type-II) diabetes mellitus, MDA: Malondialdehyde; One-way ANOVA followed by Tuceky test, $\mathrm{n}=3$. Non-significant groups: $P \geq 0.05 ;{ }^{\star} P<0.05 ;{ }^{*} P<0.001$ considered statistically significant as compared to atherosclerotic non-diabetic group.

Figure 3. Level of MDA in atherosclerotic patients with and without type 2 diabetes.

revealed that the lipid peroxidation level as an indicator of oxidative stress was significantly increased $(P<0.001)$ in diabetic patients compared to non-diabetic subjects $(0.53 \pm 0.31 \mathrm{nmol} / \mathrm{mgP}$ vs. $0.33 \pm 0.23 \mathrm{nmol} / \mathrm{mgP})$. In addition, diabetic patients had significantly lower values of TAS, GPX, SOD and Cat with respect 
to the non-diabetics group (TAS: $0.35 \pm 0.22 \mathrm{mmol} / \mathrm{l}$ vs. $0.33 \pm 0.23 \mathrm{mmol} / \mathrm{l}$, GPx: $\mathrm{U} / \mathrm{mgP} 4.27 \pm 1.76$ vs. $3.81 \pm 2.08 \mathrm{U} / \mathrm{mgP}$, SOD: $11.64 \pm 4.61 \mathrm{U} / \mathrm{mgP}$ vs. $12.433 \pm 6.11 \mathrm{U} / \mathrm{mgP}$, Cat: $1.089 \pm 0.79 \mathrm{U} / \mathrm{mgP}$ vs. $1.25 \pm 0.99 \mathrm{U} / \mathrm{mgP})$. Atherosclerotic patients with type II diabetes had slightly higher values of Se in relation to its level in non-diabetic patients $(0.039 \pm 0.0032 \mathrm{ppm}$ vs. $0.042 \pm 0.0042$ ppm) (Figure 4).

\subsection{Correlations}

Table 3 shows the correlations between TAS activity and some clinical and laboratory variables in atherosclerotic diabetic patients as well as atherosclerotic none diabetic subjects. Pearson's correlation coefficient in atherosclerotic diabetic patients was observed a significant positive correlation between TAS and HbAlc $(r=0.134, P<0.049)$ while a negative correlation was found between TAS and MDA $(r=-0.061, P<0.039)$ (Table 3$)$. In atherosclerotic non-diabetic patients, TAS showed significant negative correlation with the TG $(\mathrm{r}=-0.423, P$ $<0.006)$, MDA $(\mathrm{r}=-0.200, P<0.02)($ Table 3$)$.
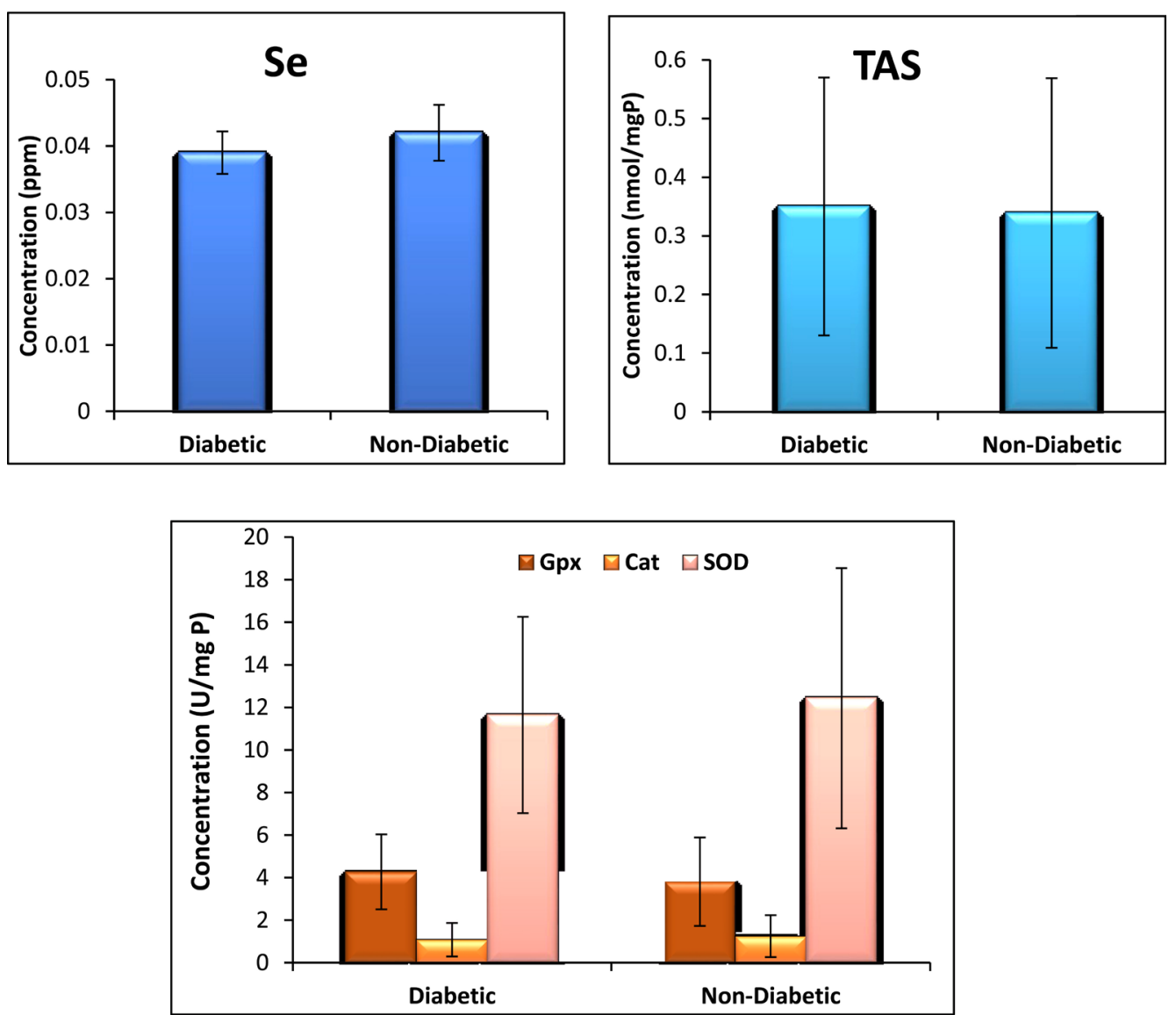

Values are expressed as mean \pm SD for $\mathrm{n}$ subjects, SOD: Superoxide dismutase; GPx: Glutathione peroxidase; Cat: Catalase. One-way ANOVA followed by Tuckey test, $\mathrm{n}=3$. Non-significant groups: $P \geq 0.05 ;{ }^{\star} P<0.05 ;{ }^{\star} P<0.001$ considered statistically significant as compared to atherosclerotic non-diabetic group.

Figure 4. Values of antioxidative parameters in atherosclerotic patients with and without type 2 diabetes. 
Table 3. Correlations of Total Antioxidants (TAS) activity with some clinical and laboratory variables in atherosclerotic patients with and without type 2 diabetes.

\begin{tabular}{ccccc}
\hline & \multicolumn{2}{c}{ Diabetic patients $(\mathbf{n}=32)$} & Non diabetic patients $(\mathbf{n}=41)$ \\
\cline { 2 - 5 } Parameters & $\mathrm{r}$ & $\mathrm{P}$ & $\mathrm{r}$ & $\mathrm{P}$ \\
\hline Age & -0.220 & 0.227 & 0.089 & 0.584 \\
BMI & -0.172 & 0.354 & -0.215 & 0.182 \\
HbA1C & 0.134 & 0.049 & -0.143 & 0.419 \\
GPX & 0.068 & 0.711 & 0.004 & 0.983 \\
SOD & -0.065 & 0.729 & 0.176 & 0.278 \\
Cat & -0.014 & 0.937 & -0.042 & 0.797 \\
HbA1c & -0.052 & 0.793 & -0.010 & 0.956 \\
HDL-C & 0.042 & 0.820 & 0.051 & 0.753 \\
LDL-C & -0.090 & 0.626 & -0.197 & 0.224 \\
TG & 0.074 & 0.686 & $-0.423 * *$ & 0.006 \\
TC & -0.025 & -0.891 & -0.261 & 0.108 \\
MDA & -0.061 & 0.039 & -0.200 & 0.021 \\
Se & 0.468 & 0.172 & 0.230 & 0.522 \\
\hline
\end{tabular}

$\mathrm{r}=$ correlation coefficient. BMI, Body mass index, ${ }^{*}$ : non-insulin dependent (Type-2) diabetes mellitus, TC: Total cholesterol; TG: Triglyceride; LDL-C: Low-density lipoprotein cholesterol; HDL-C: High-density lipoprotein cholesterol, $\mathrm{HbA}_{1 \mathrm{c}}$ : Glycosylated hemoglobin, SOD: Superoxide dismutase; GPx: Glutathione peroxidase; Cat: Catalase; MDA: Malondialdehyde. Non-significant groups: $P \geq 0.05$; ${ }^{*} P<0.05$; ${ }^{*} P<0.001$ considered statistically significant as compared to atherosclerotic non-diabetic group.

There was a correlation of GPx activity with some clinical and laboratory variables in atherosclerotic diabetic patients as well as atherosclerotic none diabetic subjects (Table 4). A significant correlation was found between GPx and SOD ( $\mathrm{r}$ $=0.359, P<0.047), \mathrm{GPx}$ and Cat $(\mathrm{r}=0.488, P<0.005), \mathrm{GPx}$ and MDA $(\mathrm{r}=$ $0.575, P<0.001)$ in atherosclerotic diabetic patients.

Atherosclerotic diabetic group, atherosclerotic non-diabetic group showed highly significant correlations between GPx and SOD ( $\mathrm{r}=0.684, P<0.000)$, GPx and Cat $(\mathrm{r}=0.488, P<0.001)$ (Table 4$)$.

In addition, Pearson's correlation reveals a positive significant correlation between MDA and GPx $(\mathrm{r}=0.575, P<0.001)$, MDA and Cat $(\mathrm{r}=0.673, P<$ $0.00)$, MDA and SOD $(\mathrm{r}=0.449, P<0.011)$ in atherosclerotic diabetic groups and in atherosclerotic non-diabetic patients, a highly significant positive correlation between MDA and Cat $(\mathrm{r}=-0.604, P<0.00)$ was obtained (Table 5).

Moreover, a significant positive correlations was obtained between SOD-GPx $(\mathrm{r}=0.359, P<0.047)$, and SOD-MDA $(\mathrm{r}=0.449, P<0.011)$ in atherosclerotic diabetics while in atherosclerotic non-diabetics as marked positive correlations were observed between SOD-GPx $(\mathrm{r}=0.684, P<0.00)$, and GPx-Cat $(\mathrm{r}=0.421$, $P<0.006$ ) (Table 6).

Furthermore, Table 7 shows the correlations between Cat activity and some 
Table 4. Correlations of Glutathione peroxidase (GPx) activity with some clinical and laboratory variables in atherosclerotic patients with and without type 2 diabetes.

\begin{tabular}{ccccc}
\hline \multirow{2}{*}{ Parameters } & \multicolumn{2}{c}{ Diabetic patients $(\mathbf{n}=\mathbf{3 2})$} & \multicolumn{2}{c}{ Non diabetic patients $(\mathbf{n}=41)$} \\
\cline { 2 - 5 } & $\mathbf{r}$ & $\mathbf{P}$ & $\mathbf{r}$ & $\mathbf{P}$ \\
\hline Age & -0.321 & 0.073 & -0.089 & 0.579 \\
BMI & -0.321 & 0.078 & 0.040 & 0.802 \\
CBC & 0.194 & 0.323 & -0.221 & 0.201 \\
TAS & 0.068 & 0.711 & 0.004 & 0.983 \\
SOD & $0.359^{*}$ & 0.047 & $0.684^{* *}$ & 0.000 \\
Cat & $0.488^{* *}$ & 0.005 & $0.489^{* *}$ & 0.001 \\
HbA1c & -0.047 & 0.814 & -0.021 & 0.901 \\
HDL-C & -0.030 & 0.870 & -0.168 & 0.294 \\
LDL-C & -0.028 & 0.879 & -0.127 & 0.430 \\
TG & 0.163 & 0.374 & -0.074 & 0.644 \\
TC & 0.011 & 0.952 & 0.004 & 0.983 \\
MDA & $0.575^{* *}$ & 0.001 & 0.275 & 0.086 \\
Se & 0.412 & 0.236 & 0.430 & 0.215 \\
\hline
\end{tabular}

$\mathrm{r}=$ correlation coefficient. BMI, Body mass index, ${ }^{*}$ : non-insulin dependent (Type-2) diabetes mellitus, TC: Total cholesterol; TG: Triglyceride; LDL-C: Low-density lipoprotein cholesterol; HDL-C: High-density lipoprotein cholesterol, $\mathrm{HbA}_{1 \mathrm{c}}$ : Glycosylated hemoglobin, SOD: Superoxide dismutase; Cat: Catalase; MDA: Malondialdehyde; TAS: Total Antioxidants. Non-significant groups: $P \geq 0.05 ;{ }^{*} P<0.05 ;{ }^{*} P<0.001$ considered statistically significant as compared to atherosclerotic non-diabetic group.

Table 5. Correlations of Malondialdehyde (MDA) activity with some clinical and laboratory variables in atherosclerotic patients with and without type 2 diabetes.

\begin{tabular}{ccccc}
\hline & \multicolumn{2}{c}{ Diabetic patients $(\mathbf{n}=\mathbf{3 2})$} & \multicolumn{2}{c}{ Non diabetic patients $(\mathbf{n}=41)$} \\
\cline { 2 - 5 } Parameters & $\mathbf{r}$ & $\mathbf{P}$ & $\mathbf{r}$ & $\mathbf{P}$ \\
\hline Age & -0.320 & 0.074 & 0.206 & 0.202 \\
BMI & -0.235 & 0.203 & -0.015 & 0.928 \\
GPx & $0.575^{* *}$ & 0.001 & 0.275 & 0.086 \\
TAS & -0.061 & 0.739 & -0.200 & 0.216 \\
SOD & $0.449^{*}$ & 0.011 & 0.155 & 0.341 \\
Cat & $0.673^{* *}$ & 0.000 & $0.604^{* *}$ & 0.000 \\
HbAlc & 0.027 & 0.894 & 0.043 & 0.805 \\
HDL-C & -0.070 & 0.704 & -0.204 & 0.207 \\
LDL-C & -0.077 & 0.677 & -0.056 & 0.733 \\
TG & -0.107 & 0.561 & 0.000 & 0.999 \\
TC & -0.126 & 0.493 & -0.143 & 0.385 \\
Se & 0.309 & 0.385 & -0.174 & 0.630 \\
\hline
\end{tabular}

$\mathrm{r}=$ correlation coefficient. BMI, Body mass index, ${ }^{*}$ : non-insulin dependent (Type-II) diabetes mellitus, TC: Total cholesterol; TG: Triglyceride; LDL-C: Low-density lipoprotein cholesterol; HDL-C: High-density lipoprotein cholesterol, HbA1c: Glycated haemoglobin $\mathrm{Al}_{\mathrm{C}}$; GPx: Glutathione peroxidase; Cat: Catalase; TAS: Total Antioxidant status. Non-significant groups: $P \geq 0.05 ;{ }^{*} P<0.05 ;{ }^{*} P<0.001$ considered statistically significant as compared to atherosclerotic non-diabetic group. 
Table 6. Correlations of Superoxide dismutase (SOD) activity with some clinical and laboratory variables in atherosclerotic patients with and without type 2 diabetes.

\begin{tabular}{ccccc}
\hline \multirow{2}{*}{ Parameters } & \multicolumn{2}{c}{ Diabetic patients $(\mathbf{n}=\mathbf{3 2})$} & \multicolumn{2}{c}{ Non diabetic patients $(\mathbf{n}=41)$} \\
\cline { 2 - 5 } & $\mathbf{r}$ & $\mathbf{P}$ & $\mathbf{r}$ & $\mathbf{P}$ \\
\hline Age & -0.099 & 0.598 & -0.147 & 0.360 \\
BMI & -0.043 & 0.822 & -0.197 & 0.218 \\
CBC & -0.114 & 0.564 & -0.242 & 0.162 \\
GPx & $0.359^{*}$ & 0.047 & $0.684^{* *}$ & .000 \\
TAS & -0.065 & 0.729 & 0.176 & 0.278 \\
MDA & $0.449^{*}$ & 0.011 & 0.155 & 0.341 \\
Cat & 0.066 & 0.726 & $0.421^{* *}$ & 0.006 \\
HbAlc & -0.020 & 0.918 & -0.050 & 0.771 \\
HDL-C & 0.082 & 0.659 & 0.061 & 0.706 \\
LDL-C & -0.230 & 0.213 & -0.101 & 0.529 \\
TG & -0.153 & 0.410 & -0.077 & 0.632 \\
TC & -0.236 & 0.202 & -0.106 & 0.514 \\
Se & 0.623 & 0.054 & 0.314 & 0.378 \\
\hline
\end{tabular}

$\mathrm{r}=$ correlation coefficient. BMI, Body mass index, ${ }^{*}$ : non-insulin dependent (Type-2) diabetes mellitus, TC: Total cholesterol; TG: Triglyceride; LDL-C: Low-density lipoprotein cholesterol; HDL-C: High-density lipoprotein cholesterol; $\mathrm{HbA}_{1 \mathrm{c}}$ : Glycosylated hemoglobin; GPx: Glutathione peroxidase; Cat: Catalase; MDA: Malondialdehyde; TAS: Total Antioxidants. Non-significant groups: $P \geq 0.05 ;{ }^{*} P<0.05 ;{ }^{*} P<0.001$ considered statistically significant as compared to atherosclerotic non-diabetic group.

clinical and laboratory variables in atherosclerotic diabetics as well as atherosclerotic non-diabetics. In atherosclerotic diabetic patients, Pearson's correlation coefficient shows significant positive correlation between Cat and GPx $(\mathrm{r}=$ $0.488, P<0.005)$ and Cat-MDA $(\mathrm{r}=0.673, P<0.00)$. In atherosclerotic non-diabetic patients Pearson's correlation observes a significant positive correlation between Cat-GPx $(\mathrm{r}=0.489, P<0.001)$, Cat-MDA $(\mathrm{r}=0.604, P<0.00)$ and Cat-SOD ( $\mathrm{r}=0.421, P<0.000)$ (Table 7).

\section{Discussion}

Accumulative evidence shows that oxidative stress plays a crucial role in the initiation and progression of a number of human diseases such as cancer, diabetes mellitus, and atherosclerosis. An imbalance between increased oxidative stress and impaired antioxidants defense may play a role in the progression of atherosclerotic disease. The present study was done to evaluate the oxidative stress parameters in the Libyans atherosclerotic participates and to demonstrate the correlation between oxidative stress, diabetes, and atherosclerosis. Present results concluded that the values of studied anti-oxidative parameters (SOD, GPx, Cat and TAS) were significantly lower in atherosclerotic participates comparatively with controls. In addition, lipid peroxidation level was significantly higher in 
Table 7. Correlations of Catalase (Cat) activity with some clinical and laboratory variables in atherosclerotic patients with and without type 2 diabetes.

\begin{tabular}{ccccc}
\hline \multirow{2}{*}{ Parameters } & \multicolumn{2}{c}{ Diabetics patients $(\mathbf{n}=32)$} & \multicolumn{2}{c}{ Non diabetics patients $(\mathbf{n}=41)$} \\
\cline { 2 - 5 } & $\mathbf{r}$ & $\mathbf{P}$ & $\mathbf{r}$ & $\mathbf{P}$ \\
\hline Age & -0.224 & 0.217 & -0.058 & 0.720 \\
BMI & -0.155 & 0.404 & -0.178 & 0.265 \\
GPx & $0.488^{* *}$ & 0.005 & $0.489^{* *}$ & 0.001 \\
TAS & -0.014 & 0.937 & -0.042 & 0.797 \\
MDA & $0.673^{* *}$ & 0.000 & $0.604^{* *}$ & 0.000 \\
SOD & 0.066 & 0.726 & $0.421^{* *}$ & 0.006 \\
HbA1c & -0.132 & 0.502 & -0.009 & 0.957 \\
HDL-C & -0.001 & 0.997 & 0.004 & 0.980 \\
LDL-C & 0.063 & 0.732 & 0.006 & 0.968 \\
TG & 0.148 & 0.420 & 0.079 & 0.625 \\
TC & 0.079 & 0.668 & 0.012 & 0.940 \\
Se & 0.197 & 0.586 & 0.029 & 0.937 \\
\hline
\end{tabular}

$\mathrm{r}=$ correlation coefficient. BMI, Body mass index, ${ }^{*}$ : non-insulin dependent (Type-2) diabetes mellitus, TC: Total cholesterol; TG: Triglyceride; LDL-C: Low-density lipoprotein cholesterol; HDL-C: High-density lipoprotein cholesterol, $\mathrm{Hb}_{1 \mathrm{c}}$ : Glycosylated hemoglobin, SOD: Superoxide dismutase; GPx: Glutathione peroxidase; MDA: Malondialdehyde; TAS: Total Antioxidants. Non-significant groups: $P \geq 0.05$; ${ }^{\star} P<0.05$; ${ }^{*} P<0.001$ considered statistically significant as compared to atherosclerotic non-diabetic group.

patients in relation to healthy subjects (Table 1).

Various studies have demonstrated that diabetes mellitus, smoking, and hypertension, consider the main risk factors of atherosclerosis, which are correlated with an increased production of ROS by the endothelium [21] [22] [23]. It has been recently found a good correlation between elevated HbAlc values and the atherosclerosis [24] [25] [26] which result from oxidative stress induced by various glucose oxidation, change in antioxidant enzymes status and impaired glutathione metabolism [27] [28].

Reactive oxygen species (ROS) especially free oxygen radicals and peroxides may have both physiological and pathological roles in atherosclerosis. ROS are important regulators of vascular homeostasis [29], they are key mediators of signaling pathways that cause vascular inflammation in atherogenesis, starting from the initiation of fatty streak development through lesion progression to ultimate plaque rupture. Animal studies found that ROS are involved in atherosclerosis by the accumulation of lipid peroxidation products and induction of inflammatory genes and activation of matrix metalloproteinases [3] [21] [30]. The findings that show TAS levels are lower and MDA levels are higher in atherosclerotic patients (diabetic and non-diabetic) as compared to the healthy group. An explanation for the observed reduction in TAS among atherosclerotic patients could be due to the presence of high amount of free radicals and other oxygen derived (superoxide) species, which ultimately activate the depletion of 
plasma antioxidants and increases lipid peroxidation [8].

ROS and reactive nitrogen species (RNS) produced by the endothelium promote oxidative modification of LDL (low-density lipoprotein). Oxidized LDL has been implicated in several facets of the inflammatory process, including the invasion of monocytes and macrophages, their differentiation, and the induction of injury to endothelial cells [31]. It has been proven that oxidized LDL involved in plaque formation in atherosclerosis as it is naturally being considered to play the most important role in lipid storage and inflammation [32] [33]. Previous studies have found that atheroscloresis is related to increase in lipid peroxidation [34] [35] [36]. An increase in lipid peroxidation product especially malondialdehyde level (MDA) may also related to the higher level of oxidized LDL. Present results correlate with previous findings [37] [38] [39] which illustrated that elevated levels of MDA indicate increase in the level of production of oxygen free radicals, suggesting their possible role in atherogenesis.

Antioxidants are a diverse group of compounds within which play important role in inhibiting oxidant formation, intercepting oxidants once they have formed, and repairing oxidant-induced injury. Although It is well documented that exposure to ROS increases the expression of antioxidant enzymes [40] [41], many studies have revealed that a decrease in the level of antioxidant defense as an expression of increase in free radical production [42] [43] [44]. Various enzyme systems including superoxide dismutase (SOD), glutathione peroxidases (GPx) and catalase (Cat) are involved in regulating ROS production and degradation in vascular cells [45] [46].

SOD, Cat and GPx play association role in the elimination of superoxide radicals, in which SOD transforms them to $\mathrm{H}_{2} \mathrm{O}_{2}$ while GPx and Cat convert $\mathrm{H}_{2} \mathrm{O}_{2}$ to water [47] [48]. Therefore, prevent the formation of hydroxyl radicals, which is considered highly toxic molecule [49] [50]. In this study, results demonstrated lower levels of SOD, GPx, and catalase in serum of atherosclerotic patients with and without diabetes as compared to controls (Figure 3 and Figure 4), that was in agreement with the outcomes of other studies [51] [52] [53]. In atherosclerotic with diabetes group, there was found a very good positive correlation between elevated MDA and decreased GPx level $(\mathrm{r}=0.575 ., P<0.001)$ (Table 5). In addition, Significant positive correlations were noted between serum MDA level and SOD activity $(\mathrm{r}=0.449, P=0.011)$ and serum MDA level and Cat activity ( $\mathrm{r}$ $=0.673, P=0.000$ ) (Table 5) which was in consistent with previous study [54]. The declined in the activity of SOD was feasibly due to the accumulation of $\mathrm{H}_{2} \mathrm{O}_{2}$, and glycation of these enzymes, which was in concord with previous findings [55] [56]. In addition, [57] were reported that the lower activity of GPx activity in atherosclerotic with diabetes group was related to inactivation of the enzyme because of severe oxidative stress condition.

Selenium, an essential micronutrient that play a pivotal role in maintaining optimal health by regulating the redox balance. It is involved in the complex system of defense against oxidative stress through selenium-dependent glutathione peroxidases and other selenoproteins. In this investigation results dem- 
onstrated that the selenium concentrations were lower in the atherosclerotic patients in relation to its content in controls which was in agreement with the outcomes of other studies [58] [59]. However, the Figure 4 shows that the distribution of patients with and without diabetes was similar at all selenium concentrations, indicating that a serum selenium is not related to the regulation and severity of the disease [17] [60].

\section{Conclusion}

An imbalance between increased oxidative stress and impaired antioxidant defense may play a role in the progression of atherosclerotic disease. Aforementioned observations indicate that oxidative stress was increased in atherosclerotic diabetic patients as approved by the significant changes in the levels of oxidative stress parameters. The relationship between oxidative stress parameters and imbalance antioxidants suggests their strong involvement in atherosclerosis. Further investigations with large clinical trials are recommended.

\section{Acknowledgements}

The authors would like to thank the medical record in charge and staff of Tajoura National Heart Center, Tripoli, Libya, for their cooperation and support during the study and patients who participate in this study.

\section{Conflicts of Interest}

The authors declare no conflicts of interest regarding the publication of this paper.

\section{Authors' Contributions}

This work was carried out in collaboration between all authors. Author RA designed the study, wrote the protocol and wrote the first draft of the manuscript. Authors SE and EA preformed the experiments, ZA collected the data and samples, author NA interpreted the analyses of the study and contributed important intellectual content, author ME performed the statistical analysis, and author AR managed the literature searches and critically revised the manuscript. All authors read and approved the final manuscript.

\section{Ethics Approval and Consent to Participate}

The Ethics committee of Biotechnology Research Center (Tripoli, Libya) approved the study (BEC-BTRC 03-2016). All patients provided a written informed consent prior to start of the study procedures.

\section{References}

[1] Pal, R.K. and Grera, A. (2017) Coronary Artery Disease in Africa: Community Based Study of Risk Factors. Bureau of Jail Management and Penology, 3, 326.

[2] Halliwell, B. and Gutteridge, J.M.C. (1999) Free Radicals in Biology and Medicine, 
3rd Edition. Oxford University Press, New York, 10-121.

[3] Bonomini, F., Tengattini, S., Fabiano, A., Bianchi, R. and Rezzani, R. (2008) Atherosclerosis and Oxidative Stress. Histology and Histopathology Cellular and Molecular Biology, 23, 381-390.

[4] Witztum, J. (1997) The Oxidation Hypothesis of Atherosclerosis. The Lancet, 344, 793-795. https://doi.org/10.1016/S0140-6736(94)92346-9

[5] Witztum, J. and Steinberg, D. (2001) The Oxidative Modification Hypothesis of Atherosclerosis Does It Hold for Humans? Trends in Cardiovascular Medicine, 11, 93-102. https://doi.org/10.1016/S1050-1738(01)00111-6

[6] Stocker, R. and Keaney, J.F. (2004) Role of Oxidative Modifications in Atherosclerosis. Physiological Reviews, 84, 1381-1478.

[7] Steinberg, D., Parthasarathy S., Carew, T.E., Khoo, J.C. and Witztum, J.L. (1989) Beyond Cholesterol. Modifications of Low-Density Lipoprotein that Increase Its Atherogenicity. The New England Journal of Medicine, 320, 915-924.

https://doi.org/10.1056/NEJM198904063201407

[8] Liaudet, L., Rosenblatt-Velin, N. and Pacher, P. (2013) Role of Peroxynitrite in the Cardiovascular Dysfunction of Septic Shock. Current Vascular Pharmacology, 11, 196-207.

[9] Incalza, M.A., D’Oria, R., Natalicchio, A., Perrini, S., Laviola, L. and Giorgino, F. (2018) Oxidative Stress and Reactive Oxygen Species in Endothelial Dysfunction Associated with Cardiovascular and Metabolic Diseases. Vascular Pharmacology, 100, 1-19. https://doi.org/10.1016/j.vph.2017.05.005

[10] Victor, V., Rocha, M., Sola, E., Banuls, C., Garcia-Malpartida, K. and Hernandez-Mijares, A. (2009) Oxidative Stress, Endothelial Dysfunction and Atherosclerosis. Current Pharmaceutical Design, 15, 2988-3002.

[11] Chen, J.Y., Ye, Z.X., Wang, X.F., Chang, J., Yang, M.W., Zhong, H.H., Hong, F.F. and Yang, S.L. (2018) Nitric Oxide Bioavailability Dysfunction Involves in Atherosclerosis. Biomedicine and Pharmacotherapy, 97, 423-428.

https://doi.org/10.1016/j.biopha.2017.10.122

[12] Katakami, N. (2017) Mechanism of Development of Atherosclerosis and Cardio-Vascular Disease in Diabetes Mellitus. Journal of Atherosclerosis and Thrombosis, 25, 27-39.

[13] Severino, P., D’Amato, A., Netti, L., Pucci, M., De Marchis, M., Palmirotta, R., Volterrani, M., Mancone, M. and Fedele, F. (2018) Diabetes Mellitus and Ischemic Heart Disease: The Role of Ion Channels. International Journal of Molecular Sciences, 19, 802. https://doi.org/10.3390/ijms19030802

[14] Pai, J.K., Cahill, L.E., Hu, F.B., Rexrode, K.M., Manson, J.E. and Rimm, E.B. (2013) Hemoglobin $A_{1 c}$ Is Associated with Increased Risk of Incident Coronary Heart Disease among Apparently Healthy, Nondiabetic Men and Women. Journal of the American Heart Association, 2, e000077. https://doi.org/10.1161/JAHA.112.000077

[15] Hjalmarsson, C., Manhem, K., Bokemark, L. and Andersson, B. (2014) The Role of Prestroke Glycemic Control on Severity and Outcome of Acute Ischemic Stroke. Stroke Research and Treatment, 2014, Article ID: 694569. https://doi.org/10.1155/2014/694569

[16] Taleb, A., Ahmad, K.A., Ihsan, A.U., Qu, J., Lin, N., Hezam, K., Koju, N., Hui, L. and Dong, Q. (2018) Antioxidant Effects and Mechanism of Silymarin in Oxidative Stress Induced Cardiovascular Diseases. Biomedicine \& Pharmacotherapy, 102, 689-698. https://doi.org/10.1016/j.biopha.2018.03.140 
[17] Kornhauser, C., Garcia-Ramirez, J.R., Wrobe, K., Perez-Luque, E.L., Garay-Sevilla, M.E. and Wrobe, K. (2008) Serum Selenium and Glutathione Peroxidase Concentrations in Type 2 Diabetes Mellitus Patients. Primary Care Diabetes, 2, 81-85. https://doi.org/10.1016/j.pcd.2008.02.003

[18] Zhang, Y.-T., Zheng, Q.-S., Pan, J. and Zheng, R.-L. (2004) Oxidative Damage of Biomolecules in Mouse Liver Induced by Morphine and Protected by Antioxidants. Basic \& Clinical Pharmacology \& Toxicology, 95, 53-58. https://doi.org/10.1111/j.1742-7843.2004.950202.x

[19] Hadwan, M.H. (2016) New Method for Assessment of Serum Catalase Activity. Indian Journal of Science and Technology, 9, 1-5. https://doi.org/10.17485/ijst/2016/v9i4/80499

[20] Hafeman, D.G., Sunde, R.A. and Hoekstra, W.G. (1974). Effect of Dietary Selenium on Erythrocyte and Liver Glutathione Peroxidase in the Rat. The Journal of Nutrition, 104, 580-587. https://doi.org/10.1093/jn/104.5.580

[21] Forbes, J.M. and Cooper, M.E. (2013) Mechanisms of Diabetic Complications. Physiological Reviews, 93, 137-188.

[22] Fan, L.M., Douglas, G., Bendall, J.K., McNeill, E., Crabtree, M.J., Hale, A.B., Jian-Mei Li, A.M., McAteer, M.A., Schneider, J.E., Choudhury, R.P. and Channon, K.M. (2014) Endothelial Cell-Specific Reactive Oxygen Species Production Increases Susceptibility to Aortic Dissection. Circulation, 129, 2661-2672. https://doi.org/10.1161/CIRCULATIONAHA.113.005062

[23] Yuan, T., Yang, T., Chen, H., Fu, D., Hu, Y., Wang, J., Yuan, Q., Yu, H., Xu, W. and Xie, X. (2019) New Insights into Oxidative Stress and Inflammation during Diabetes Mellitus-Accelerated Atherosclerosis. Redox Biology, 20, 247-260.

https://doi.org/10.1016/j.redox.2018.09.025

[24] Xing, F.Y., Neeland, I.J., Gore, M.O., Ayers, C.R., Paixao, A.R., Turer, A.T., Berry, J.D., Khera, A., de Lemos, J.A. and McGuire, D.K. (2014) Association of Prediabetes By Fasting Glucose and/or Haemoglobin $A_{1 c}$ Levels with Subclinical Atherosclerosis and Impaired Renal Function: Observations from the Dallas Heart Study. Diabetes and Vascular Disease Research, 11, 11-18. https://doi.org/10.1177/1479164113514239

[25] Fernández-Friera, L., Fuster, V., López-Melgar, B., Oliva, B., García-Ruiz, J.M., Mendiguren, J., Bueno, H., Pocock, S., Ibáñez, B., Fernández-Ortiz, A. and Sanz, J. (2017) Normal LDL-Cholesterol Levels Are Associated with Subclinical Atherosclerosis in the Absence of Risk Factors. Journal of the American College of Cardiology, 70, 2979-2991. https://doi.org/10.1016/j.jacc.2017.10.024

[26] Nazish, S., Zafar, A., Shahid, R., Albakr, A., Alkhamis, F.A., Aljaafari, D., Alabdali, M., Alsulaiman, A. and Al-Mulla, F.A. (2018) Relationship between Glycated Haemoglobin and Carotid Atherosclerotic Disease among Patients with Acute Ischaemic Stroke. Sultan Qaboos University Medical Journal, 18, 311-317.

[27] Butterfield, D.A., Domenico, F. and Di.and Barone, E. (2014) Elevated Risk of Type 2 Diabetes for Development of Alzheimer Disease: A Key Role for Oxidative Stress in Brain. Biochimica et Biophysica Acta (BBA)_Molecular Basis of Disease, 1842, 1693-1706. https://doi.org/10.1016/j.bbadis.2014.06.010

[28] Nguyen, D., Hsu, J.W., Jahoor, F. and Sekhar, R.V. (2014) Effect of Increasing Glutathione with Cysteine and Glycine Supplementation on Mitochondrial Fuel Oxidation, Insulin Sensitivity, and Body Composition in Older HIV Infected Patients. The Journal of Clinical Endocrinology and Metabolism, 99, 169-177. https://doi.org/10.1210/jc.2013-2376 
[29] Chen, Q., Wang, Q., Zhu, J., Xiao, Q. and Zhang, L. (2017) Reactive Oxygen Species: Key Regulators in Vascular Health and Diseases. British Journal of Pharmacology, 175, 1279-1292. https://doi.org/10.1111/bph.13828

[30] Heymes C, Bendall, J.K., Ratajczak, P., Cave, A.C., Samuel, J.L., Hasenfuss, G. and Shah, A.M. (2003) Increased Myocardial NADPH Oxidase Activity in Human Heart Failure. Journal of the American College of Cardiology, 41, 2164-2171. https://doi.org/10.1016/S0735-1097(03)00471-6

[31] Yamada, N. (2001) Atherosclerosis and Oxidative Stress. Japan Medical Association Journal, 44, 529-534.

[32] Uchida, K. (2015) Aldehyde Adducts Generated during Lipid Peroxidation Modification of Proteins. Free Radical Research, 49, 896-904.

https://doi.org/10.3109/10715762.2015.1036052

[33] Orekhov, A.N. and Myasoedova, V.A. (2019) Low Density Lipoprotein-Induced Lipid Accumulation Is a Key Phenomenon of Atherogenesis at the Arterial Cell Level. Vessel Plus, 3, 14. https://doi.org/10.20517/2574-1209.2018.80

[34] Amaki, T., Suzuki, T., Nakamura, F., Hayashi, D., Imai, Y., Morita, H., Fukino, K., Nojiri, T., Kitano, S., Hibi, N., Yamazaki, T. and Nagai, R. (2004) Circulating Malondialdehyde Modified LDL Is a Biochemical Risk Marker for Coronary Artery Disease. Heart, 90, 1211-1213. https://doi.org/10.1136/hrt.2003.018226

[35] Polidori, M.C., Pratico, D., Parente, B., Mariani, E., Cecchetti, R., Yao, Y., Sies, H., Cao, P., Mecocci, P. and Stahl, W. (2007) Elevated Lipid Peroxidation Biomarkers and Low Antioxidant Status in Atherosclerotic Patients with Increased Carotid or Iliofemoral Intima Media Thickness. Journal of Investigative Medicine, 55, 163-167. https://doi.org/10.2310/6650.2007.06043

[36] Vogiatzi, G., Tousoulis, D. and Christodoulos, S. (2009) The Role of Oxidative Stress in Atherosclerosis Oxidative Stress and Atherosclerosis. Hellenic Journal of Cardiology, 50, 402-409.

[37] Yang, R.L., Shi, Y.H., Hao, G., Li, W. and Le, G.W. (2008) Increasing Oxidative Stress with Progressive Hyperlipidemia in Human: Relation between Malon-Dialdehyde and Atherogenic Index. Journal of Clinical Biochemistry and Nutrition, 43, 154-158. https://doi.org/10.3164/jcbn.2008044

[38] Viigimaa, M., Abina, J., Zemtsovskaya, G., Tikhaze, A., Konovalova, G., Kumskova, E. and Lankin, V. (2010) Malondialdehyde-Modified Low-Density Lipoproteins as Biomarker for Atherosclerosis. Blood Pressure, 19, 164-168. https://doi.org/10.3109/08037051.2010.484158

[39] Stanek, A., Cholewka, A., Wielkoszyński, T., Romuk, E., Sieroń, K. and Sieroń, A. (2017) Increased Levels of Oxidative Stress Markers, Soluble CD40 Ligand, and Carotid Intima-Media Thickness Reflect Acceleration of Atherosclerosis in Male $\mathrm{Pa}$ tients with Ankylosing Spondylitis in Active Phase and without the Classical Cardiovascular Risk Factors. Oxidative Medicine and Cellular Longevity, 2017, Article ID: 9712536. https://doi.org/10.1155/2017/9712536

[40] Worthington Enzyme Manual (2009) Worthington Biochemical Corporation. http://www.worthington-biochem.com/index/manual.html

[41] Ray, P.D. Huang, B.W. and Tsuji, Y. (2012) Reactive Oxygen Species (ROS) Homeostasis and Redox Regulation in Cellular Signaling. Cellular Signalling, 24, 981-990. https://doi.org/10.1016/j.cellsig.2012.01.008

[42] Singh, Z., Karthigesu, I.P., Singh, P. and Kaur R. (2014) Use of Malondialdehyde as a Biomarker for Assessing Oxidative Stress in Different Disease Pathologies: A Review. Iranian Journal of Public Health, 43, 7-16. 
[43] Milanlioglu, A., Aslan, M., Ozkol, H., Çilingir, V., Nuri Aydın, M. and Karadas, S. (2016) Serum Antioxidant Enzymes Activities and Oxidative Stress Levels in Patients with Acute Ischemic Stroke: Influence on Neurological Status and Outcome. Wiener Klinische Wochenschrift, 128, 169-174. https://doi.org/10.1007/s00508-015-0742-6

[44] Ziaadini, F., Aminae, M., Rastegar, M.M., Abbasian, S. and Memari, A.H. (2017) Melatonin Supplementation Decreases Aerobic Exercise Training Induced-Lipid Peroxidation and Malondialdehyde in Sedentary Young Women. Polish Journal of Food and Nutrition Sciences, 67, 225-232. https://doi.org/10.1515/pjfns-2017-0001

[45] Papaharalambus, M.C.A. and Griendling, K.K. (2007) Basic Mechanisms of Oxidative Stress and Reactive Oxygen Species in Cardiovascular Injury. Trends in Cardiovascular Medicine, 17, 48-54. https://doi.org/10.1016/j.tcm.2006.11.005

[46] Lü, J.M., Lin, P.H., Yao, Q. and Chen, C. (2010) Chemical and Molecular Mechanisms of Antioxidants: Experimental Approaches and Model Systems. Journal of Cellular and Molecular Medicine, 14, 840-860. https://doi.org/10.1111/j.1582-4934.2009.00897.x

[47] Dal, S. and Sigrist, S. (2016) The Protective Effect of Antioxidants Consumption on Diabetes and Vascular Complications. Diseases, 4, 24. https://doi.org/10.3390/diseases4030024

[48] Kwon, I., Jang, Y., Song, W., Roltsch, M.H. and Lee, Y. (2018) Mitochondrial Antioxidant Enzymes and Endurance Exercise-Induced Cardioprotection against Ischemia-Reperfusion Injury. Sports and Exercise Medicine Open Journal, 4, 9-15. https://doi.org/10.17140/SEMOJ-4-155

[49] Styskal, J., van Remmen, H., Richardson, A. and Salmon, A.B. (2012) Oxidative Stress and Diabetes: What Can We Learn about Insulin Resistance from Antioxidant Mutant Mouse Models? Free Radical Biology and Medicine, 52, 46-58. https://doi.org/10.1016/j.freeradbiomed.2011.10.441

[50] Li, H., Horke, S. and Förstermann, U. (2013) Oxidative Stress in Vascular Disease and Its Pharmacological Prevention. Trends in Pharmacological Sciences, 34, 313-319. https://doi.org/10.1016/j.tips.2013.03.007

[51] Zawadzka-Bartczak, E. (2005) Activities of Red Blood Cell Anti-Oxidative Enzymes (SOD, GPx) and Total Anti-Oxidative Capacity of Serum (TAS) in Men with Coronary Atherosclerosis and in Healthy Pilots. Medical Science Monitor, 11, 440-444.

[52] Čolak, E., Dimitrijević-Srećković, V., Djordjević, R.B., Stanković, S., Glišić1, B., koSrećković, B. and Majkic-Singh, N. (2008) Biomarkers of Enzymatic and Nonenzymatic Antioxidative Defense in Type 2 Diabetes Mellitus-Comparative Analysis. Biochemia Medica, 18, 42-51. https://doi.org/10.11613/BM.2008.006

[53] Chehaibi, K., Trabelsi, I., Mahdouani, K. and Slimane, M.N. (2016) Correlation of Oxidative Stress Parameters and Inflammatory Markers in Ischemic Stroke Patients. Journal of Stroke and Cerebrovascular Diseases, 25, 2585-2593. https://doi.org/10.1016/j.jstrokecerebrovasdis.2016.06.042

[54] Abou-Seif, M.A. and Youssef, A.A. (2004) Evaluation of Some Biochemical Changes in Diabetic Patients. Clinica Chimica Acta, 346, 161-170. https://doi.org/10.1016/j.cccn.2004.03.030

[55] Matough, F.A., Budin, S.B., Hamid, Z.A., Alwahaibi, N. and Mohamed, J. (2012) The Role of Oxidative Stress and Antioxidants in Diabetic Complications. Sultan Qaboos University Medical Journal, 12, 5-18.

[56] Briggs, O.N., Brown, H., Elechi-amadi, K., Ezeiruaku, F. and Nduka, N. (2016) Superoxide Dismutase and Glutathione Peroxidase Levels in Patients with Long 
Standing Type 2 Diabetes in Port Harcourt, Rivers State, Nigeria. International Journal of Science and Research, 5, 1282-1288.

https://doi.org/10.21275/v4i5.NOV162149

[57] Condell, R.A. and Tapell, A.L. (1983) Evidence for Suitability of Glutathione Peroxidase as a Protective Enzyme: Studies of Oxidative Damage, Restoration and Proteolysis. Archives of Biochemistry and Biophysics, 223, 407-416.

[58] Sanmartin, C., Plano, D., Font, M. and Palop, J.A. (2011) Selenium and Clinical Trials: New Therapeutic Evidence for Multiple Diseases. Current Medicinal Chemistry, 18, 4635-4650. https://doi.org/10.2174/092986711797379249

[59] Liang, J., Gao, Y., Tang, S., Feng, S., Han, J. and Hu, Q. (2018) Serum Levels of Trace Elements and Vitamins in Coronary Artery Disease and Their Predictive Values. International Journal of Clinical and Experimental Medicine, 11, 922-931.

[60] Kljai, K. and Runje, R. (2001) Selenium and Glycogen Levels in Diabetic Patients. Biological Trace Element Research, 83, 223-229.

https://doi.org/10.1385/BTER:83:3:223 